\title{
Audit of post-exposure treatment to prevent lyssavirus infection in Sydney South West Area Health Service, 2005-2007
}

\section{Adam T. Craig ${ }^{\mathrm{A}, \mathrm{C}}$, Trish F. Mannes ${ }^{\mathrm{B}}$ and Leena Gupta ${ }^{\mathrm{B}}$}

ANSW Public Health Officer Training Program, NSW Department of Health

BPublic Health Unit, Sydney South West Area Health Service

${ }^{\mathrm{C}}$ Corresponding author. Email:

adam.craig@doh.health.nsw.gov.au

\begin{abstract}
Objectives: To describe the profile of people who received post-exposure treatment to prevent lyssavirus infection in Sydney South West Area Health Service between 2005 and 2007 and to assess treatment compliance with the current NSW Health protocol. Methods: Thirty-eight public health files and a subset of 11 medical records were reviewed to collect demographic, exposure and treatment information for the period. Results: Twenty-nine (76\%) potential Lyssavirus exposures occurred overseas. Nine potential exposures occurred within Australia; eight of these resulted from a bat bite or scratch. Thirteen (34\%) of all potential exposures resulted from handling an animal. Conclusion: Many potential exposures were the result of a bite or scratch from a domesticated animal; the animal's survival or health status was not routinely recorded. While all people who commenced post-exposure treatment completed the prescribed course, this was often not within the stipulated timeframe.
\end{abstract}

Lyssavirus is a zoonotic viral disease that generally infects domestic and wild mammals. The genus Lyssavirus falls within the family Rhabdoviridae. There are seven genotypes recognised within the genus (although a number of other viruses await classification). These are rabies virus; Lagos bat virus; Mokola virus; Duvenhage virus; the European bat lyssaviruses 1 and 2; and Australian bat lyssavirus (ABLV). ${ }^{1-3}$
Lyssavirus is typically transmitted to humans in the virusladen saliva of an infected animal through a bite, scratch or lick on broken skin or mucous membranes. ${ }^{1-3}$ Symptoms of lyssavirus infection include: headache; fever; malaise; sensory change around the site of the bite or scratch; weakness; excitability; aversion to air and water; delirium; convulsions; and coma. Once symptoms of lyssavirus infection develop, death usually follows within 2 to 6 days. $^{3}$ An estimated 55000 deaths per year are caused by lyssavirus genotype 1 (rabies) worldwide, mostly in rural areas of Asia and Africa. Asia carries the largest public health burden, with an estimated 24000 deaths per year. ${ }^{4}$

While lyssavirus genotype 1 (rabies) has never been reported in Australia, ABLV has emerged as an important reservoir for the disease. ABLV rarely infects humans and only two cases of human infection have been recorded in Australia. Both cases occurred in the mid 1990s and were fatal. ${ }^{5-7}$ People who handle Australian fruit bats (Pteropus sp.) are at risk of contracting $\mathrm{ABLV}$, whereas those who come into contact with unvaccinated domestic or wild animals in countries where lyssavirus 1 is endemic are at risk of contracting rabies. ${ }^{3}$ In more than $99 \%$ of all human lyssavirus 1 cases worldwide, the virus has been transmitted from a dog. ${ }^{8}$

The most effective mechanism to aid in the protection against Lyssavirus is to wash the wound site immediately for a minimum of 15 minutes with soap or detergent and water, followed by the application of an antiviral antiseptic such as ethanol $(70 \%)$, iodine or providone iodine. ${ }^{3,9-11}$ For people not previously vaccinated against Lyssavirus, post-exposure treatment consists of five intramuscular injections of $1 \mathrm{~mL}$ rabies vaccine at days $0,3,7,14$ and 28-30, and one dose of human rabies immunoglobulin (HRIG) at the time of the first post-exposure vaccine. It is recommended that as much of the HRIG as possible be infiltrated into the wound site. For people who have had previous vaccination against Lyssavirus, post-exposure treatment consists of two doses of vaccine at day 0 and day $3 \cdot^{3,8,9,12}$

In New South Wales (NSW), public health units are responsible for authorising all post-exposure treatment, in accordance with the Rabies and Other Lyssavirus Infections Response Protocol for NSW Public Health 
Units. $^{3}$ This paper describes the profile of people who received post-exposure treatment in Sydney South West Area Health Service (SSWAHS) between 2005 and 2007, and assesses if treatment was delivered in accordance with the current NSW Health protocol.

\section{Methods}

We extracted the public health records for notifications of potential exposure to Lyssavirus received by the SSWAHS Public Health Unit between 1 January 2005 and 31 May 2007, and collected de-identified patient demographic information and exposure data. One-third of the extracted files were randomly selected and the patients' medical records were accessed from either their general practitioners or the hospitals that provided the post-exposure treatment. We collected information from the medical records about the nature of the exposure and adherence to treatment. We verified the information collected from the public health records. We were unable to obtain medical records for two cases. Data was extracted by AC (author) and collated and analysed using Microsoft Excel.

The audit was approved by the human research ethics committee of Royal Prince Alfred Hospital.

\section{Results}

From 1 January 2005 to 31 May 2007, the SSWAHS Public Health Unit received notifications and recommended post-exposure treatment for 38 people (18 males and 20 females), potentially exposed to Lyssavirus. Seventeen of these were for people potentially exposed in 2005, 17 in 2006 and four in the first 5 months of 2007. Fifty-three percent were aged between 21 and 40 years.

Of the 38 people who sought post-exposure treatment, 29 (76\%) were potential exposures while overseas and nine (24\%) while in Australia. Of the 29 potential overseas exposures, 12 were exposed in Thailand; three in China and two each in India, Vietnam, Malaysia and the Philippines. Five of the remaining six people were potentially exposed in South-East Asian countries.

Dog bites $(n=15,54 \%)$ and dog scratches $(n=5,15 \%)$ were the most common form of potential overseas exposure. Other potential overseas exposures were associated with bites or scratches from monkeys ( $n=6,21 \%)$, cats $(n=2,7 \%)$ and one mouse $(3 \%)$. The World Health Organization does not recommend post-exposure treatment of potential exposure to rabies associated with mouse bite or scratch. ${ }^{13}$ Bites or scratches from domesticated animals accounted for $16(55 \%)$ potential overseas exposures; 13 of these were from domesticated dogs. Nondomestic animals accounted for 12 (41\%) potential overseas exposures; six from wild dogs and four from wild monkeys (Table 1). Of those potentially exposed while overseas, six (21\%) people reported that they had handled the animal that bit or scratched them.

Of the nine potential exposures occurring in Australia, eight were the result of a wild bat bite or scratch and one bite was from a vaccinated dog imported from Italy.

Seven (78\%) of the people potentially exposed in Australia reported that they handled the animal that caused the injury. The injuries occurred when handling, releasing, detangling or assisting a bat (Table 1). No records were available about the health of the animal in the days following the potential exposure and no records indicated that any of the animals were tested for Lyssavirus.

Of the 11 randomly selected medical records reviewed to assess compliance with post-exposure treatment, three people commenced post-exposure treatment on the day of exposure, three within 7 days of exposure, one on the eighth day and one 33 days after potential exposure. The three other records reported commencement of treatment while overseas and within a week of potential exposure. All 11 people completed the full five-dose course of postexposure treatment; five within the 30-day period stipulated in the Response Protocol for NSW Public Health Units, three within 40 days of exposure and three completed the course more than 50 days after exposure. ${ }^{3}$ Three people received HRIG within a week of exposure, three did not receive HRIG at all and no record was available to determine if HRIG was provided to the other five patients.

\section{Discussion}

This audit provides a useful description of people receiving post-exposure treatment to prevent lyssavirus infection in SSWAHS and supports the findings of similar work undertaken by Young et al. in Queensland. ${ }^{14}$ The audit was able to identify risk behaviours that contribute to potential exposure to Lyssavirus, both in Australia and overseas. Of note is the number of people $(n=13,34 \%)$ who were exposed when handling the animal that bit or scratched them, in particular people exposed to bat bites or scratches in Australia $(n=5,56 \%)$. Simple strategies to mitigate this exposure, such as not engaging with animals or use of personal protective equipment in occupational settings, may reduce the incidence of potential exposure.

Fifty percent of all exposures were a result of bite or scratch from a domesticated animal. As an animal's survival (or health status) is a clear indicator of infection, the collection of this information can inform the risk assessment and subsequent treatment, particularly for those seeking post-exposure treatment weeks after being potentially exposed. The World Health Organization recommends ceasing post-exposure treatment if the animal remains healthy throughout an observation period of 10 days or if the animal is euthanised and found to be negative for 
Table 1. Demographic and exposure details of people potentially exposed overseas and in Australia to rabies/lyssavirus in Sydney South West Area Health Service, Jan 2005 to May 2007

\begin{tabular}{|c|c|c|c|c|c|c|}
\hline & \multicolumn{2}{|c|}{$\begin{array}{c}\text { Potentially exposed } \\
\text { overseas }\end{array}$} & \multicolumn{2}{|c|}{$\begin{array}{c}\text { Potentially exposed in } \\
\text { Australia }\end{array}$} & \multicolumn{2}{|c|}{ Total } \\
\hline & $N$ & $\%$ & $N$ & $\%$ & $N$ & $\%$ \\
\hline \multicolumn{7}{|l|}{ Demographics } \\
\hline Male & 16 & 55 & 2 & 22 & 18 & 47 \\
\hline Female & 13 & 45 & 7 & 78 & 20 & 53 \\
\hline Persons & 29 & 100 & 9 & 100 & 38 & 100 \\
\hline \multicolumn{7}{|l|}{ Age } \\
\hline $0-20$ & 2 & 7 & 1 & 11 & 3 & 8 \\
\hline $21-40$ & 17 & 59 & 3 & 33 & 20 & 53 \\
\hline $41-60$ & 5 & 17 & 2 & 22 & 7 & 18 \\
\hline $61-80$ & 4 & 14 & 1 & 11 & 5 & 13 \\
\hline$>80$ & 1 & 3 & 1 & 11 & 2 & 5 \\
\hline \multicolumn{7}{|l|}{ Animal } \\
\hline Dog & 20 & 69 & $1^{*}$ & 11 & 21 & 55 \\
\hline Bat & 0 & 0 & 8 & 89 & 8 & 21 \\
\hline Monkey & 6 & 21 & 0 & 0 & 6 & 16 \\
\hline Cat & 2 & 7 & 0 & 0 & 2 & 5 \\
\hline Mouse & 1 & 3 & 0 & 0 & 1 & 3 \\
\hline \multicolumn{7}{|l|}{ Domestication of animal } \\
\hline Non-domestic & 12 & 41 & 8 & 89 & 20 & 53 \\
\hline Domestic & 16 & 55 & 1 & 11 & 17 & 45 \\
\hline Unknown & 1 & 3 & 0 & 0 & 1 & 3 \\
\hline \multicolumn{7}{|l|}{ Wound type } \\
\hline Bite & 18 & 62 & 5 & 56 & 23 & 61 \\
\hline Scratch & 6 & 21 & 3 & 33 & 9 & 24 \\
\hline Unknown & 5 & 17 & 1 & 11 & 6 & 16 \\
\hline \multicolumn{7}{|l|}{ Engaged with animal } \\
\hline Yes & 6 & 21 & 7 & 78 & 13 & 34 \\
\hline No & 22 & 76 & 1 & 11 & 23 & 61 \\
\hline Unknown & 1 & 3 & 1 & 11 & 2 & 5 \\
\hline \multicolumn{7}{|l|}{ First aid at time of exposure } \\
\hline Cleaned with soap/water & 18 & 62 & 7 & 78 & 26 & 68 \\
\hline Cleaned at health care facility & 6 & 21 & 0 & 0 & 6 & 16 \\
\hline None administered & 2 & 7 & 2 & 22 & 4 & 11 \\
\hline Unknown & 3 & 10 & 0 & 0 & 3 & 8 \\
\hline
\end{tabular}

Data source: Sydney South West Public Health Unit files and patients' medical records.

*Potentially exposed from an imported canine.

rabies. ${ }^{9}$ For potential exposures from bats in Australia, national guidelines state that where possible the bat should be kept and tested for Lyssavirus at an appropriate reference laboratory. ${ }^{10}$

People who sought post-exposure treatment for Lyssavirus generally did so soon after being exposed and completed the course of treatment, but not always within the timeframe stipulated in the NSW Health protocol. Emphasis on adherence to treatment and mechanisms to follow up patients and health-care providers to facilitate timely delivery of treatment may be required to improve compliance with the protocol in the future.
The value of this audit is limited by the small number of cases available to review and the quality of information in medical and public health records about the treatment rationale, follow-up, previous treatment (if post-exposure treatment commenced elsewhere) or the factors contributing to the potential exposure. Due to these limitations, we note that the findings of the audit should be interpreted with caution and recommend its replication in other area health services or on a statewide basis to gain a better understanding of the issue. In addition, our study only reflects circumstances where post-exposure treatment was requested and does not include potentially exposed people who did not seek treatment. 


\section{Conclusion}

This study used routinely collected data to review potential exposures to Lyssavirus and compliance with the NSW Health treatment protocol for people seeking post-exposure treatment in SSWAHS. The audit identified that: many potential exposures to Lyssavirus were the result of a bite or scratch from a domesticated animal; an animal's survival or health status at the time of the potential exposure or afterwards is not routinely recorded; and all people who commenced post-exposure treatment completed the prescribed course, although not always within the timeframe stipulated in the protocol.

\section{References}

1. NSW Health. Rabies and bat lyssavirus infection. N SW Public Health Bull 2002; 13(1-2): 27.

2. World Health Organization Media Centre. Fact Sheet - Rabies. Geneva: World Health Organization; 2008. Available from: http://www.who.int/mediacentre/factsheets/fs099/en/ (Cited 14 November 2008.)

3. NSW Health. Rabies and other lyssavirus infections: response protocol for NSW Public Health Units, Notifiable Diseases Manual. Sydney: NSW Health; July 2007. Available from: http://www.health.nsw.gov.au/factsheets/guideline/rabies.html (Cited 14 November 2008.)

4. WHO Expert. Consultation on Rabies: First Report. WHO Technical Report Series 931. Geneva: World Health Organization; 2005. Available from: http://www.who.int/rabies/ trs931_\%2006_05.pdf (Cited 14 November 2008.)

5. Hanna J, Carney I, Smith G, Tannenberg A, Deverill J, Botha J et al. Australian Bat Lyssavius infection: a second human case with long incubation period. Med J Aust 2000; 172(12): 573-4.
6. Allworth A, Murray K, Morgan J. A human case of encephalitis due to a lyssavirus recently identified in fruit bats. $C D I$ 1996; 20(24): 504.

7. NSW Health. Infectious Disease Factsheet. Rabies and Bat Lyssavirus Infection. Sydney: NSW Health; 2008. Available from: http://www.health.nsw.gov.au/factsheets/infectious/ rabiesbatinfection.html (Cited 14 November 2008.)

8. National Health and Medical Research Council. The Australian Immunisation Handbook. 9th edition. Canberra: NHMRC and Australian Government Department of Health and Ageing; 2008. pp. 110-7.

9. World Health Organization. WHO Recommendations on Rabies Post-Exposure Treatment and the Correct Technique of Intradermal Immunization Against Rabies. Geneva: WHO; 1996. Available from: http://whqlibdoc.who.int/hq/1996/ WHO_EMC_ZOO_96.6.pdf (Cited 14 November 2008.)

10. Commonwealth Department of Health and Ageing. Australian Bat Lyssavirus Information for Medical Practitioners. Canberra: Commonwealth Department of Health and Ageing; 2001.

11. Rabies vaccine-update. Wkly Epidemiol Rec 2007; 82(8): $62-8$.

12. Wilde H. Failure of post exposure rabies prophylaxis. Vaccine 2007; 25(44): 7605-9. doi:10.1016/j.vaccine.2007.08.054

13. World Organization for Animal Health. Health Standards. Manual of Diagnostic Tests and Vaccines for Terrestrial Animals. Chapter 2.2.5. Rabies. Paris: World Organization for Animal Health; 2007. Available from: http://www.oie.int/Eng/ Normes/Mmanual/A_00044.htm (Cited 14 November 2008.)

14. Young M, McCall B. Trends in potential exposure to Australian bat lyssavirus in South East Queensland, 1996 to 2003.

Commun Dis Intell 2004; 28(2): 258-60. 